\title{
Regression Verification using Impact Summaries
}

\author{
John Backes ${ }^{1}$, Suzette Person ${ }^{2}$, Neha Rungta ${ }^{3}$, and Oksana Tkachuk ${ }^{3}$ \\ 1 University of Minnesota \\ back0145@umn.edu \\ 2 NASA Langley Research Center \\ suzette.person@nasa.gov \\ 3 NASA Ames Research Center \\ neha.s.rungta, oksana.tkachuk@nasa.gov
}

\begin{abstract}
Regression verification techniques are used to prove equivalence of closely related program versions. Existing regression verification techniques leverage the similarities between program versions to help improve analysis scalability by using abstraction and decomposition techniques. These techniques are sound but not complete. In this work, we propose an alternative technique to improve scalability of regression verification that leverages change impact information to partition program execution behaviors. Program behaviors in each version are partitioned into (a) behaviors impacted by the changes and (b) behaviors not impacted (unimpacted) by the changes. Our approach uses a combination of static analysis and symbolic execution to generate summaries of program behaviors impacted by the differences. We show in this work that checking equivalence of behaviors in two program versions reduces to checking equivalence of just the impacted behaviors. We prove that our approach is both sound and complete for sequential programs, with respect to the depth bound of symbolic execution; furthermore, our approach can be used with existing approaches to better leverage the similarities between program versions and improve analysis scalability. We evaluate our technique on a set of sequential $\mathrm{C}$ artifacts and present preliminary results.
\end{abstract}

\section{Introduction}

Various reduction, abstraction, and compositional techniques have been developed to help scale software verification techniques to industrial-sized systems. Although such techniques have greatly increased the size and complexity of systems that can be checked, analysis of large software systems remains costly. Regression analysis techniques, e.g., regression testing [15], regression model checking [22], and regression verification [19], restrict the scope of the analysis by leveraging the differences between program versions. These techniques are based on the idea that if code is checked early in development, then subsequent versions can be checked against a prior (checked) version, leveraging the results of the previous analysis to reduce analysis cost of the current version.

Regression verification addresses the problem of proving equivalence of closely related program versions [19]. These techniques compare two programs with a 
large degree of syntactic similarity to prove that portions of one program version are equivalent to the other. Regression verification can be used for guaranteeing backward compatibility, and for showing behavioral equivalence in programs with syntactic differences, e.g., when a program is refactored to improve its performance, maintainability, or readability.

Existing regression verification techniques leverage similarities between program versions by using abstraction and decomposition techniques to improve scalability of the analysis $[9,11,19]$. The abstractions and decomposition in the these techniques, e.g., summaries of unchanged code [11] or semantically equivalent methods [19], compute an over-approximation of the program behaviors. The equivalence checking results of these techniques are sound but not completethey may characterize programs as not functionally equivalent when, in fact, they are equivalent.

In this work we describe a novel approach that leverages the impact of the differences between two programs for scaling regression verification. We partition program behaviors of each version into (a) behaviors impacted by the changes and (b) behaviors not impacted (unimpacted) by the changes. Only the impacted program behaviors are used during equivalence checking. We then prove that checking equivalence of the impacted program behaviors is equivalent to checking equivalence of all program behaviors for a given depth bound. In this work we use symbolic execution to generate the program behaviors and leverage controland data-dependence information to facilitate the partitioning of program behaviors. The impacted program behaviors are termed as impact summaries. The dependence analyses that facilitate the generation of the impact summaries, we believe, could be used in conjunction with other abstraction and decomposition based approaches, [9,11], as a complementary reduction technique. An evaluation of our regression verification technique shows that our approach is capable of leveraging similarities between program versions to reduce the size of the queries and the time required to check for logical equivalence.

The main contributions of this work are:

- A regression verification technique to generate impact summaries that can be checked for functional equivalence using an off-the-shelf decision procedure.

- A proof that our approach is sound and complete with respect to the depth bound of symbolic execution.

- An implementation of our technique using the LLVM compiler infrastructure, the KLEE Symbolic Virtual Machine [3], and a variety of Satisfiability Modulo Theory (SMT) solvers, e.g., STP [6] and Z3 [5].

- An empirical evaluation on a set of $\mathrm{C}$ artifacts which shows that the use of impact summaries can reduce the cost of regression verification.

\section{Motivation and Background}

\subsection{Checking Functional Equivalence}

In this work, we focus on functional equivalence [11]. Two programs, $P_{0}$ and $P_{1}$, are functionally equivalent iff for all possible input values to the programs, they 


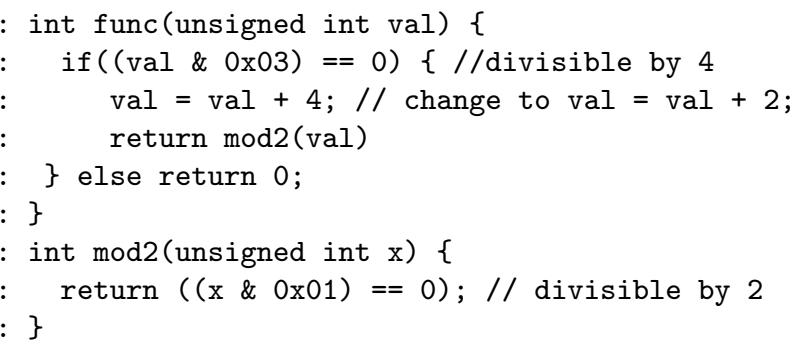

Fig. 1. Program behavior is unchanged when the constant value in line 3 is even.

both produce the same output, i.e., they return the same value and result in the same global state. In general, proving functional equivalence is undecidable, so we prove functional equivalence with respect to a user-specified depth-bound for loops and recursive functions. Note that this notion of equivalence is similar to the k-equivalence defined in [19].

Equivalence checking techniques that use uninterpreted functions as a mechanism for abstraction and decomposition $[9,11,19]$ produce sound but not complete results. The example in Figure 1 demonstrates how the use of uninterpreted functions can lead to false negatives. The input to methods func and mod2 is an unsigned integer. If the input to func, val, is divisible by four, then in version $V_{0}$ of func, four is added to val and method mod2 is invoked with the updated variable, val. Next, mod2 returns true if its input, $x$, is divisible by two; otherwise it returns false. Suppose, a change is made to line 3 in $V_{1}$ of fun and two is added to val in lieu of four. Both versions of func are functionally equivalent, i.e., for all possible inputs to func, the output is the same in both versions.

Symdiff is a technique which uses uninterpreted functions during equivalence checking [9]. It modularly checks equivalence of each pair of procedures in two versions of the program. To check the equivalence of the func method, it replaces the call to mod2 at line 4 with an uninterpreted function. The inputs to the uninterpreted function are parameters and global values read by the method. In $V_{0}$ of func the uninterpreted function for the call to mod2 is $f_{-}$mod2 (val + 4) while in $V_{1}$ it is $f \_m o d 2(v a l+2)$. The procedures are then transformed to a single logical formula whose validity is checked using verification condition generation. Symdiff will report $V_{0}$ and $V_{1}$ of func as not equivalent due to the different input values to the uninterpreted function: $f_{-} \bmod 2$. The use of uninterpreted functions results in an over-approximation because equality logic with uninterpreted functions (EUF) relies on functional congruence (consistency) - a conservative approach to judging functional equivalence which assumes that instances of the same function return the same value if given equal arguments [8]. Other equivalence checking techniques that rely on uninterpreted functions will report similar false negatives. 
1: int $\mathrm{a}, \mathrm{b}$;

2: void test(int $x$, int $y)\{$

3: if $(x>0) a=a+1$; else $a=a+2 ; / /$ change $x<=0$

4: if $(y>0) b=b+1$; else $b=b+2$;

5: $\}$

Fig. 2. An example where equivalence cannot be naively checked using DiSE.

\subsection{Symbolic Execution}

Symbolic execution uses symbolic values in lieu of concrete values for program inputs and builds a path condition for each execution path it explores. A path condition contains (a) a conjunction of constraints over the symbolic input values and constants such that they represent the semantics of the statements executed on a given path $p$ and (b) the conjunction of constraints that represent the effects of executing $p$ - the return value and the final global state. The disjunction of all the path conditions generated during symbolic execution is a symbolic summary of the program behaviors. Version $V_{0}$ of the test method in Figure 2 has two integer inputs $x$ and $y$ whose values determine the updates made to the global variables $a$ and $b$. There are four path conditions for $V_{0}$ generated by symbolic execution:

1. $x>0 \wedge y>0 \wedge a_{0}=a+1 \wedge b_{0}=b+1$

2. $\neg(x>0) \wedge y>0 \wedge a_{1}=a+2 \wedge b_{0}=b+1$

3. $x>0 \wedge \neg(y>0) \wedge a_{0}=a+1 \wedge b_{1}=b+2$

4. $\neg(x>0) \wedge \neg(y>0) \wedge a_{1}=a+2 \wedge b_{1}=b+2$.

Each path condition has constraints on the inputs $x$ and $y$ that lead to the update of global variables $a$ and $b$. The variables $a_{0}, a_{1}, b_{0}$, and $b_{1}$ are temporary variables that represent the final assignments to global variables $a$ and $b$.

\subsection{Change Impact Analysis}

The DiSE framework, in our previous work, implements a symbolic execution based change impact analysis for a given software maintenance task $[12,16]$. DiSE uses the results of static change impact analyses to direct symbolic execution toward the parts of the code that may be impacted by the changes. The output of DiSE is a set of impacted path conditions, i.e., path conditions along program locations impacted by differences in programs.

The inputs to DiSE are two program versions and a target client analysis. DiSE first computes a syntactic diff of the program versions to identify locations in the source code that are modified. Then DiSE uses program slicing-based techniques to detect impacted program locations, i.e., locations that have controland data-dependencies on the modified program locations. The set of impacted program locations is used to direct symbolic execution to explore execution paths 
containing impacted locations. In the parts of the program composed of locations not impacted by the change, DiSE explores a subset of the feasible paths through that section.

The dependence analyses and pruning within the DiSE framework are configurable based on the needs of the client analysis. To illustrate how DiSE computes path conditions for generating test inputs to cover impacted branch statements, consider the example in Figure 2. Suppose a change is made to line 3 where the condition $x>0$ in $V_{0}$ of test is changed to $x<=0$ in $V_{1}$. Due to this change, the conditional statement and assignments to global variable $a$ on line 3 are marked as impacted in both versions. The goal of the symbolic execution in DiSE is to generate path conditions that cover both true and false branches of the conditional branch statement, $x<=0$, and explore any one of the branches of the conditional branch statement, $y>0$. The path conditions for program version, $V_{0}$, that may be generated by DiSE are:

1. $x>0 \wedge y>0 \wedge a_{0}=a+1 \wedge b_{0}=b+1$

2. $\neg(x>0) \wedge y>0 \wedge a_{1}=a+2 \wedge b_{0}=b+1$;

Here both branches of the $x \leq 0$ are explored while the true branch of the $y>0$ is explored. Similarly the path conditions for version $V_{1}$ that may be generated by DiSE are:

1. $x \leq 0 \wedge \neg(y>0) \wedge a_{0}=a+1 \wedge b_{1}=b+2$

2. $\neg(x \leq 0) \wedge \neg(y>0) \wedge a_{1}=a+2 \wedge b_{1}=b+2$.

In version, $V_{1}$ both branches of $x \leq 0$ are still explored but the false branch of the $y>0$ is explored. Note this is because DiSE does not enforce a specific branch to be explored for an unimpacted conditional statement. These path conditions can be solved to generate test inputs that drive execution along the paths that contain impacted locations.

The path conditions generated for regression testing, related to impacted branch coverage, in the DiSE framework under-approximate the program behaviors. As shown above the constraints on the variable $y$ in the path conditions generated by DiSE shown above are different in $V_{0}$ from those generated in $V_{1}$ - the path conditions represent different under-approximations of the program behaviors. This under-approximation does not allow the path conditions to be used for equivalence checking. Furthermore the dependence analysis is also tailored to suit the needs of the client analyses. The client analyses that are currently supported in DiSE are related to regression testing (test inputs to satisfy different coverage criteria) and improving DARWIN based delta debugging.

In this work we add support for performing equivalence checking within the DiSE framework. For this we define a set of static change impact rules that allow us to precisely characterize the program statements as impacted or unimpacted such that checking equivalence of behaviors of two programs reduces to the problem of checking equivalence of the behaviors encoded by the impacted statements. 


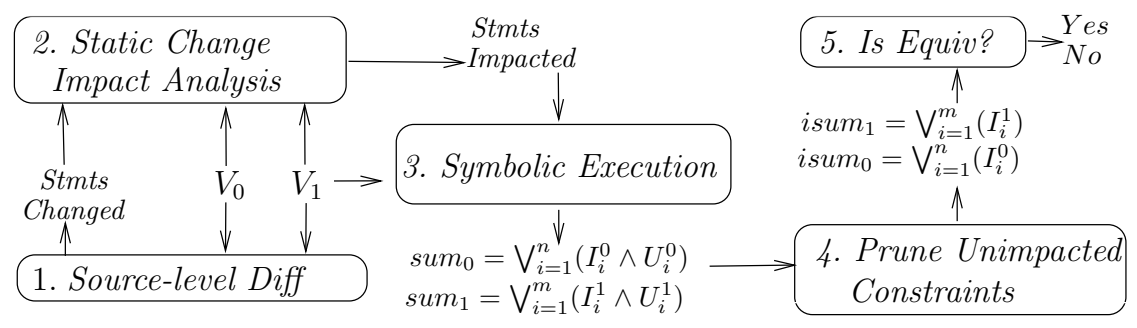

Fig. 3. Overview of regression verification using impact summaries.

\section{Regression Verification using Impact Summaries}

An overview of our regression verification technique is shown in Figure 3. Steps 1-3 in Figure 3 represent a static change impact analysis that is performed on $V_{0}$ and $V_{1}$. The change impact analysis marks the program statements that are impacted by the differences between $V_{0}$ and $V_{1}$. The outputs from Step 3 are the program behavior summaries (full summaries) for program versions $V_{0}$ and $V_{1}$. Each symbolic summary consists of the path conditions representing the program execution behaviors.

In order to facilitate the characterization of the program behaviors as impacted or unimpacted, we first define a mechanism to distinguish between different behaviors encoded within a given path condition. For the example shown in Figure 2 each path condition encodes two program behaviors; the first program behavior is related to the input variable, $x$, and global variable, $a$; while the second program behavior is related to the input variable $y$ and global variable $b$. We can make this distinction because the operations on variables $x$ and $a$ are completely disjoint from the operations on variables $y$ and $b$. The constraints on $x$ and $a$ represent one set of program behaviors for the example in Figure 2 while the constraints on $y$ and $b$ represent another set of behaviors. Based on this distinction a path condition can contain num behaviors such that the set of constraints encoding each behavior are completely disjoint from the constraints encoding the other behaviors.

In this work, we partition the constraints in each path condition generated by the change impact analysis as either impacted or unimpacted. An impacted (unimpacted) constraint $I_{i}\left(U_{i}\right)$ is a constraint that is added to the path condition as a result of executing an impacted (unimpacted) program statement during symbolic execution. The conjunction of the impacted constraints, $I_{i}$, in a path condition represents impacted program behaviors, while the conjunction of the unimpacted constraints, $U_{i}$ in a path condition, represents unimpacted program behaviors. 
(1) if $S_{i} \in \mathbb{I}$ and $S_{j}$ is control dependent on $S_{i}$ then $\mathbb{I} \cup\left\{S_{j}\right\}$

(2) if $S_{i} \in \mathbb{I}$ and $S_{j}$ uses (reads) the value of a variable defined (written) at $S_{i}$ then $\mathbb{I} \cup\left\{S_{j}\right\}$

(3) if $S_{i} \in \mathbb{I}$ an $S_{i}$ is control dependent on $S_{j}$ then $\mathbb{I} \cup\left\{S_{j}\right\}$

(4) if $S_{i} \in \mathbb{I}$ and $S_{j}$ defines (writes) a variable whose value is used (read) at $S_{i}$ then $\mathbb{I} \cup\left\{S_{j}\right\}$

Table 1. Control and data dependence rules for computing impacted statements.

Definition 1. A full summary is a disjunction of the impacted constraints $I_{i}$ and the unimpacted constraints $U_{i}$ for a program with $n$ paths: sum $=\bigvee_{i=1}^{n}\left(I_{i} \wedge\right.$ $\left.U_{i}\right)$.

For example, the full summary for $V_{0}$ containing $n$ paths is given by $\operatorname{sum}_{0}=$ $\bigvee_{i=1}^{n}\left(I_{i}^{0} \wedge U_{i}^{0}\right)$. The full summaries are post-processed in Step 4, as shown in Figure 3 to remove the unimpacted constraints and generate impact summaries.

Definition 2. An impact summary consists of a disjunction of the impacted constraints $I_{i}$ for a program with $n$ paths: isum $=\bigvee_{i=1}^{n}\left(I_{i}\right)$.

The resulting impact summaries are then checked for functional equivalence [11] in Step 5, by using an off-the-shelf SMT solver, e.g., STP [6] or Z3 [5] to check for logical equivalence. In Section 4 we prove that the full summaries for two programs are functionally equivalent iff their impact summaries are functionally equivalent.

$$
\left[\left(\bigvee_{i=1}^{n} I_{i}^{0}\right) \leftrightarrow\left(\bigvee_{i=1}^{m} I_{i}^{1}\right)\right] \leftrightarrow\left[\bigvee_{i=1}^{n}\left(I_{i}^{0} \wedge U_{i}^{0}\right) \leftrightarrow \bigvee_{i=1}^{m}\left(I_{i}^{1} \wedge U_{i}^{1}\right)\right]
$$

\subsection{Computing Impacted Program Statements and Behaviors}

In this section we present the set of rules that are necessary to conservatively compute, for sequential programs, the set of program statements that may be impacted by added or deleted program statements. We then briefly discuss how the set of impacted statements can be used to compute impacted program behaviors. The static analysis in this work uses standard control- and data-flow analysis to compute the set of impacted statements. The rules for the forward and backward flow analysis are shown in Table 1. Given the conservative nature of the analysis, it may mark certain unimpacted statements as impacted. The analysis, however, is guaranteed to find all impacted statements. We present a high-level description of how the rules are applied in the steps below:

Step 1 A source-level syntactic diff is performed to generate the change sets for the related program versions $V_{0}$ and $V_{1}$. The change set for $V_{0}$ is $\mathbb{C}_{0}$. It contains the set of statements in $V_{0}$ that are removed in $V_{1}$. The change set for $V_{1}$ is $\mathbb{C}_{1}$ which contains statements in $V_{1}$ that are added with respect to $V_{0}$. Note that all edited statements can be treated as removed in one version and added in another. 
Step 2 The impact set for program version $V_{0}$ is initialized with statements in the change set of $V_{0}: \mathbb{I}_{0}:=\mathbb{C}_{0}$.

Step 3 To account for forward control- and data-flow dependence, rules (1) and (2) in Table 1 are iteratively applied to $\mathbb{I}_{0}$ until they reach a fixpoint.

Step 4 The impact set for program version $V_{1}$ is initialized to the change set of $V_{1}: \mathbb{I}_{1}:=\mathbb{C}_{1}$.

Step 5 For all statements in the impact set of $V_{0}, \forall S_{i} \in \mathbb{I}_{0}$, if there exists a corresponding statement $S_{i}^{\prime} \in V_{1}$ such that $S_{i} \sim S_{i}^{\prime}$-then it is added to the impact set of $V_{1}, \mathbb{I}_{1}:=\mathbb{I}_{1} \cup\left\{S_{i}^{\prime}\right\}$. This step is performed to account for the impact of the statements removed in $V_{0}$.

Step 6 To compute the impact of the changes using forward control- and data-flow dependences, rules (1) and (2) in Table 1 are iteratively applied to $\mathbb{I}_{1}$ until a fixpoint is reached. Rule (3) is applied once to $\mathbb{I}_{1}$ to account for backward control-flow dependence. Finally, Rule (4) is applied to $\mathbb{I}_{1}$ transitively to compute the reaching definitions.

Step 7 Statements from the impact set of $V_{1}$ are mapped to the impact set of $V_{0}: \forall S_{i} \in \mathbb{I}_{1}$, if there exists a corresponding statement in $S_{i}^{\prime} \in V_{0}, S_{i} \sim S_{i}^{\prime}$ - then it is added to the impact set of $V_{0}, \mathbb{I}_{0}:=\mathbb{I}_{0} \cup\left\{S_{i}^{\prime}\right\}$. This step accounts for the impact of statements added to $V_{1}$.

The constraints generated by symbolic execution at impacted program statements on path $i$ are added to the impact summary, $I_{i}$ while the unimpacted constraints are added to $U_{i}$. We can check functional equivalence of two programs using their impact summaries.

The static analysis rules presented in this section compute the set of impacted program statements within a method, i.e., the analysis is intraprocedural. In [16] we present an interprocedural change impact analysis. The algorithm in [16] statically computes the impacted program statements (impact set) for all the methods disregarding the flow of impact through different method invocations. During symbolic execution these impact sets are then dynamically refined based on the calling context, propagating the impact of changes between methods through method arguments, global variables and method return values. Due to space limitations we present only the intraprocedural version of the impact analysis in this paper. Our empirical evaluation of regression verification, however, is performed using the interprocedural version of the algorithm. Next we present an example to illustrate how impact summaries are computed for an interprocedural program.

\subsection{Example}

Figure 4 shows two versions of the $\mathrm{C}$ function Init_Data that invoke the same function Set_Struct. Note that even though the analysis is performed on the single static assignment form of the program, to enable better readability we describe it in terms of the source. The Init_Data function first initializes two arrays, Data0 and Data1, and the pointer to a data structure, StructA. Then, if the value of capacity is greater than the constant length defined for arrays Data0 or Data1, the function returns zero; otherwise, it returns one. $V_{1}$ is a 


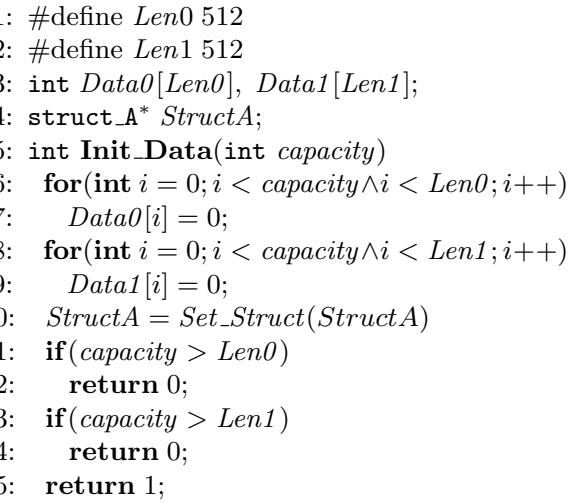

(a) $V_{0}$
1: \#define Len 0512

int Data0 [LenO], Data1[LenO];

: struct_A* StructA;

: int Init_Data(int capacity)

for $($ int $i=0 ; i<$ capacity $\wedge i<\operatorname{Len} 0 ; i++)$

$\operatorname{Data0}[i]=0$;

$\operatorname{Data1}[i]=0$;

Struct $A=$ Set_Struct $($ Struct $A)$

if $($ capacity $>$ LenO $)$

return 0 ;

return 1;

(b) $V_{1}$

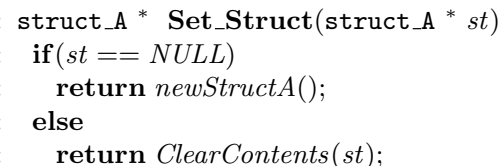

Fig. 4. Two related versions of Init_Data that are functionally equivalent.

refactored version of $V_{0}$. In $V_{1}$, a single value specifies the length of both arrays, Data0 and Data1. The refactoring also moves the initialization of Data1 into the upper for loop. The two versions of Init_Data in Figure 4 are functionally equivalent; given same value of capacity, both implementations produce the same output, i.e., return the same value, and Data0, Data1, and StructA will point to the same initialized memory ${ }^{4}$.

The edits to the refactored program version in Figure 4 are related to statements that access and edit the array Data1 and the constant Len1. These edits, however, do not impact the program statements that reference the data structure StructA and Data0. First, let us consider the accesses to StructA (via st in function Set_Struct); these are completely disjoint from the operations related to Data1 and Len1. Hence, the program behaviors related to the operations on st in this context are not impacted by the changes. The constraints related to StructA and st generated at line 10 in $V_{0}$ and line 8 in $V_{1}$ of function Init_Data and at lines $2-5$ in function Set_Struct are unimpacted and can safely be discarded from the full summaries before checking equivalence. Now, consider the accesses to Data0 and its interactions with accesses to Data1. Although the assignments to both Data0 and Data1 are control dependent on the for loop at line 6 , in the context of $V_{0}$, the assignment to Data0 is not impacted by the changes. Consequently, the constraints on Data0 at line 7 can also be discarded before checking equivalence. Moreover, functional equivalence of $V_{0}$ and $V_{1}$ in Figure 4 can be proven using impact summaries that do not contain constraints over Data0, StructA, or st.

The arrays Data0 and Data1, the pointer to StructA, and the input variable capacity are defined as symbolic in this example. In Table 2(a) we show a

${ }^{4}$ We make no claims about the initialized memory's location (the value of the pointers), only the contents of the memory. 


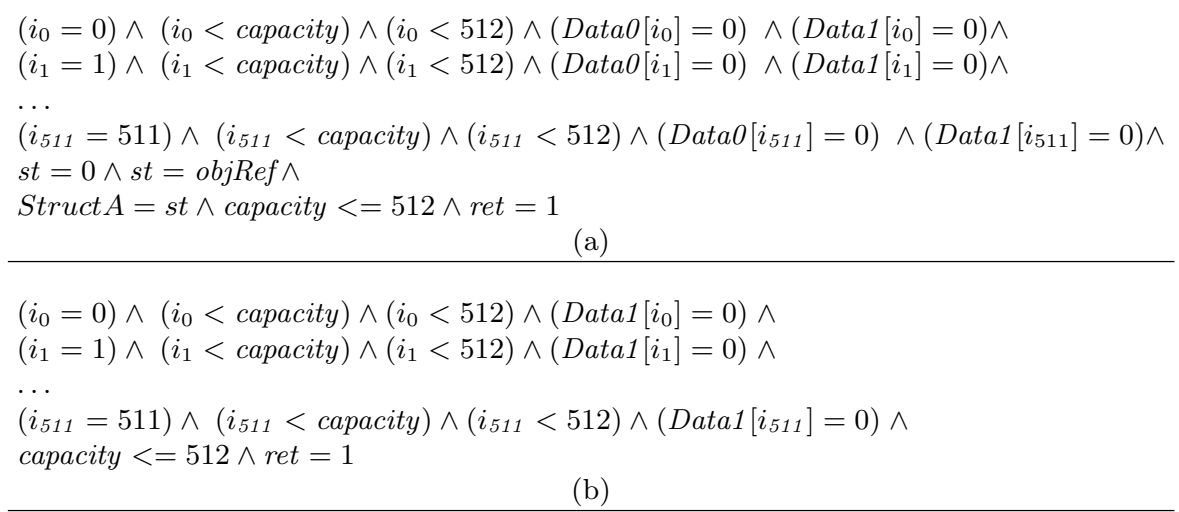

Table 2. (a) A conjunction of an unimpacted and impacted constraints along path $i$ in $V_{1}: I_{i}^{1} \bigwedge U_{i}^{1}$. (b) An impacted constraint along path $i$ in $V_{1}: I_{i}^{1}$.

summary for the path in program $V_{1}$ shown in Figure 4(b) that contains both impacted and unimpacted constraints. There are 512 iterations of the for loop that are encoded in the path using the loop index $i$, and there are constraints over StructA, st, and capacity as well. Whereas, in Table 2(b) we show only the set of impacted constraints from the same path. From this example, we can see that discarding unimpacted constraints can dramatically reduce the size of the summaries used in regression verification.

\section{Correctness Proofs}

In this section we compare two program versions $V_{0}$ and $V_{1}$. We eventually show that the equivalence of their respective summaries, sum 0 and $s u m_{1}$, can be implied by proving the equivalence of $i s u m_{0}$ and $i s u m_{1}$. Likewise, we show that if isum $_{0}$ and isum $_{1}$ are not equivalent, then sum $_{0}$ and sum su $_{1}$ are not equivalent.

To simplify the presentation of our work, we discuss the correctness of the equivalence checking using the intraprocedural change impact analysis. The same correctness argument holds for an interprocedural analysis that dynamically tracks the flow of impact through method parameters and global variables. The change impact analysis described in Section 3 is conservative for sequential programs; it adds every statement that may be impacted by a change to the impact sets $\mathbb{I}_{0}$ and $\mathbb{I}_{1}$. We argue that the statements that are considered unimpacted by the analysis are not relevant to a proof of equivalence of the program versions.

Lemma 1. Given closely related program versions $V_{0}$ and $V_{1}$, if a program statement is common to both versions, then it is either impacted in both versions or unimpacted in both versions.

Proof. This follows from Steps 5 and 7 of the static impact analysis shown in Table 1 (Section 3). Step 5 assigns $\mathbb{I}_{1}$ to be equal to $\mathbb{I}_{0}$ after performing the data- 
and control-flow analysis on $V_{0}$ (except for statements that are removed in $V_{1}$ or added in $V_{0}$ ). Then Step 7 adds statements from $\mathbb{I}_{1}$ to $\mathbb{I}_{0}$ after performing the data-flow, control-flow, backward control-flow, and reaching definition analysis on $V_{1}$ (except for statements added to $V_{1}$ or removed from $V_{0}$ ). Therefore, the only statements that differ between $\mathbb{I}_{0}$ and $\mathbb{I}_{1}$ are those that have been added or removed.

Next we argue that for every path $i$ in $V_{0}$, there exists a path $j$ in $V_{1}$ such that $i$ and $j$ contain the same set of unimpacted statements and, similarly, for every path $j$ in $V_{1}$, there exists a path $i$ in $V_{0}$ such that $i$ and $j$ contain the same set of unimpacted statements.

Lemma 2. Given closely related program versions $V_{0}$ and $V_{1}$, for every path $\left(I_{i}^{0} \wedge U_{i}^{0}\right)$ there exists a path $\left(I_{j}^{1} \wedge U_{j}^{1}\right)$ such that $U_{i}^{0} \equiv U_{j}^{1}$. Likewise, for every path $\left(I_{j}^{1} \wedge U_{j}^{1}\right)$ there exists a path $\left(I_{i}^{0} \wedge U_{i}^{0}\right)$ such that $U_{j}^{1} \equiv U_{i}^{0}$

Proof. By contradiction. Assume there is some path containing a certain sequence of unimpacted instructions in one program version but not the other. This implies that the result of some conditional statement $S_{c}$ differs between the two versions and that the set of unimpacted instructions is control dependent on $S_{c}$. Clearly the predicate in $S_{c}$ uses the result of an impacted write statement or $S_{c}$ is control dependent on another impacted conditional statement. According to Rules (1) - (4) in Table 1, $S_{c}$ is impacted. Furthermore, because the unimpacted statements are control dependent on $S_{c}$, they are also impacted.

Corollary 1. The set of unique unimpacted constraints in $V_{0}$ is the same as the set of unique unimpacted constraints in $V_{1}$. This implies Formula 2

$$
\left(\bigvee_{i=1}^{n} U_{i}^{0}\right) \leftrightarrow\left(\bigvee_{i=1}^{m} U_{i}^{1}\right)
$$

As defined in Section 3, a program's symbolic summary consists of the disjunction of the constraints along each possible execution path in the program. Each path consists of a set of impacted and unimpacted constraints. In Theorem 1 we show that the unimpacted and impacted constraints can be effectively de-coupled from each other in a program's summary.

Theorem 1. Given a program version $V_{0}$ with $n$ paths, Formula 3 is valid.

$$
\bigvee_{i=1}^{n}\left(I_{i}^{0} \wedge U_{i}^{0}\right) \leftrightarrow\left[\left(\bigvee_{i=1}^{n} I_{i}^{0}\right) \wedge\left(\bigvee_{i=1}^{n} U_{i}^{0}\right)\right]
$$

Proof. See Section A in the appendix for this proof. 
In Theorem 2 we consider the overlap between the space of assignments to program variables that satisfy impacted constraints and the space of assignments to program variables that satisfy unimpacted constraints. Specifically, we claim that for some path in a program summary, if there is some concrete assignment to the program variables that satisfies the impacted constraints, then there is a concrete assignment to the remaining variables (those only present in the unimpacted constraints) that satisfies the unimpacted constraints.

Theorem 2. Consider a program version $V_{0}$ with $n$ paths and a closely related program version $V_{1}$ with $m$ paths. Let $u_{1}, u_{2}, \ldots u_{k}$ be program variables present in the unimpacted statements of $V_{0}\left(V_{1}\right)$. Let $A U$ be the set of possible concrete assignments to these variables. Let $A I_{0}\left(A I_{1}\right)$ be the set of possible concrete assignments to all other variables in $V_{0}\left(V_{1}\right)$. For any assignment $x \in A I_{0}$ $\left(x \in A I_{1}\right)$ that satisfies the impacted constraints, there exists an assignment $y \in A U$ that satisfies the unimpacted constraints. Formally, Formulas 4 and 5 are valid.

$$
\begin{aligned}
& \forall_{x \in A I_{0}} \exists_{y \in A U}\left(I_{i}^{0}[x] \rightarrow U_{i}^{0}[y]\right) \\
& \forall_{x \in A I_{1}} \exists_{y \in A U}\left(I_{i}^{1}[x] \rightarrow U_{i}^{1}[y]\right)
\end{aligned}
$$

Proof. Rule (4) in Table 1 dictates that the statements defining the value of every variable used in an impacted statement are also impacted. Accordingly, the variables that are common to the impacted and unimpacted statements are not constrained by the unimpacted statements. I.e., the result of an unimpacted statement cannot affect the result of an impacted statement. Therefore, if it is possible to satisfy the constraints of $I_{i}^{0}\left(I_{j}^{1}\right)$, then it is possible to satisfy the constraints of $U_{i}^{0}\left(U_{j}^{1}\right)$.

Now we show that the impact summaries for two programs versions $V_{0}$ and $V_{1}$ are equivalent if and only if the summaries for $V_{0}$ and $V_{1}$ are equivalent. We use the result of Theorem 1 to prove the forward direction (if the impact summaries are equivalent, then the summaries are equivalent). Then we use the result of Theorem 2 to prove the reverse direction (if the summaries are equivalent, then the impact summaries are equivalent).

Theorem 3. Given program version $V_{0}$ with $n$ paths and a closely related program version $V_{1}$ with $m$ paths. isum $_{0}$ and isum $_{1}$ are equivalent if and only if sum $_{0}$ and sum s $_{1}$ are equivalent. This is formally stated in Formula 1 and is also shown below.

$$
\left[\left(\bigvee_{i=1}^{n} I_{i}^{0}\right) \leftrightarrow\left(\bigvee_{i=1}^{m} I_{i}^{1}\right)\right] \leftrightarrow\left[\bigvee_{i=1}^{n}\left(I_{i}^{0} \wedge U_{i}^{0}\right) \leftrightarrow \bigvee_{i=1}^{m}\left(I_{i}^{1} \wedge U_{i}^{1}\right)\right]
$$

Proof. $(\Rightarrow)$ We begin by assuming Formula 6 is valid

$$
\left(\bigvee_{i=1}^{n} I_{i}^{0}\right) \leftrightarrow\left(\bigvee_{i=1}^{m} I_{i}^{1}\right)
$$


Conjoining the term representing the disjunction of unimpacted constraints of $V_{0}$ to the left and right side of Formula 6 yields Formula 7.

$$
\left(\bigvee_{i=1}^{n} I_{i}^{0}\right) \wedge\left(\bigvee_{i=1}^{n} U_{i}^{0}\right) \leftrightarrow\left(\bigvee_{i=1}^{m} I_{i}^{1}\right) \wedge\left(\bigvee_{i=1}^{n} U_{i}^{0}\right)
$$

Applying Formula 2 yields Formula 8.

$$
\left(\bigvee_{i=1}^{n} I_{i}^{0}\right) \wedge\left(\bigvee_{i=1}^{n} U_{i}^{0}\right) \leftrightarrow\left(\bigvee_{i=1}^{m} I_{i}^{1}\right) \wedge\left(\bigvee_{i=1}^{m} U_{i}^{1}\right)
$$

Applying Formula 3 yields Formula 9.

$$
\bigvee_{i=1}^{n}\left(I_{i}^{0} \wedge U_{i}^{0}\right) \leftrightarrow \bigvee_{i=1}^{m}\left(I_{i}^{1} \wedge U_{i}^{1}\right)
$$

This proves the forward direction, i.e., $\left(\right.$ isum $_{0} \leftrightarrow$ isum $\left._{1}\right) \rightarrow\left(\right.$ sum $_{0} \leftrightarrow$ sum $\left._{1}\right)$. The latter half of the proof, $\left(\right.$ sum $_{0} \leftrightarrow$ sum $\left._{1}\right) \rightarrow\left(\right.$ isum $_{0} \leftrightarrow$ isum $\left._{1}\right)$, is more complex than the first half and is available in Appendix B.

\section{Evaluation}

To empirically evaluate the regression verification technique described in this work, we implemented a DiSE framework, Proteus, for analyzing C programs. Note that the earlier DiSE framework implementation was an extension of the Java PathFinder, [21], toolkit to analyze Java programs [12,16]. A large number of safety critical systems are developed in C; Proteus was developed at NASA to assist in the analysis of these systems.

In Proteus, we use the GNU DiffUtils ${ }^{5}$ to compute the initial change set containing the actual source level differences between program versions. The static analysis is implemented as a customized LLVM optimization pass [10]. The output of the static analysis is the set of impacted program statements. The partitioning of constraints during symbolic execution is implemented as an extension to the KLEE symbolic execution engine [3]. As an optimization for discarding unimpacted constraints, we employ the directed search in the DiSE algorithm to prune execution of paths that differ only in unimpacted constraints $[12,16]$. The final post-processing of the symbolic summaries is performed using a custom application that iterates over the impacted path conditions, removing constraints that are not impacted by the differences. We use the Z3 constraint solver to check for logical equivalence of impact summaries [5].

We present the results for the different versions of the six artifacts in Table 3. The details of the artifacts and their versions are described in Appendix C. The experiments are run on a 64 -bit Linux machine, with a $2.4 \mathrm{GHz}$ processor, and $64 \mathrm{~GB}$ memory. The Example column lists the name of the artifact and the

\footnotetext{
${ }^{5}$ http://www.gnu.org/software/diffutils
} 


\begin{tabular}{|c|c|c|c|c|c|c|c|c|c|c|c|c|}
\hline Example & Versions & Equiv & \multicolumn{2}{|c|}{\begin{tabular}{|c|} 
Paths \\
Full|iDiSE
\end{tabular}} & \multicolumn{3}{|c|}{ Constraints } & \multicolumn{2}{|c|}{\begin{tabular}{|c}
$\mid$ Time Symbc \\
Full iDiSE
\end{tabular}} & $\begin{array}{r}\mathrm{Ti} \\
\text { Full }\end{array}$ & me So & $\begin{array}{l}\text { ver } \\
\text { iSum }\end{array}$ \\
\hline nit_Data & 0V1 & yes & 400 & 400 & 103400 & 103400 & 82800 & 51.87 & 50.67 & 1.94 & 1.94 & 0.76 \\
\hline tcas1 & & & & 12 & & 524 & 332 & & & & 0.04 & \\
\hline & & & 118 & 118 & 4772 & 4772 & 3956 & & 92 & 09 & 0.09 & .06 \\
\hline & & & 118 & 118 & 4796 & 4796 & 2908 & 1.02 & 1.91 & 0.08 & 0.08 & U. \\
\hline tcas2 & $0 \mathrm{~V} 1$ & 10 & 150 & 12 & 6052 & 520 & 328 & 2.21 & 0.63 & 0.12 & 0.06 & 0.0 \\
\hline replace & & yes & 18 & 8 & 98 & 68 & 48 & 0.3 & 0.25 & 0.01 & 0.03 & 0.0 \\
\hline & & & 18 & 10 & 98 & 98 & 78 & & .32 & & 0.04 & .0 \\
\hline & & 110 & 18 & 2 & 98 & 8 & 4 & & & & .03 & 0.0 \\
\hline replace 2 & $\mathrm{~V} 1 \mathrm{~V} 2$ & yes & 604 & 604 & 23736 & 23736 & 20980 & 1.14 & 1.35 & 0.11 & 0.11 & 0.10 \\
\hline wbs1 & & & & 190 & 416 & & & & & & & \\
\hline & & yes & 336 & 336 & & & & & & & & \\
\hline & & yes & 336 & 190 & 3416 & 1478 & 10784 & 1 & 34 & .11 & 0.09 & $0.0 \varepsilon$ \\
\hline wbs2 & & no & 336 & 134 & 13388 & 5601 & 4551 & 1.18 & 0.83 & 0.11 & 0.06 & 0.06 \\
\hline cornell1 & & & 10 & & 62 & 48 & 24 & & & & 0.03 & 0.0 \\
\hline cornell2 & & yes & 18 & 10 & 1864 & 810 & 663 & 0.27 & 0.29 & 0.01 & 0.01 & 0.01 \\
\hline kernel1 & & & & & & 282 & 226 & & 21.0 & & 218 & 20 \\
\hline & & & & & & 282 & 226 & & 21.32 & & 211 & 20 \\
\hline & & & 4 & 2 & 130 & 114 & 88 & 1.56 & 1.92 & 0.20 & 0.13 & 0.0 \\
\hline kernel3 & V0V1 & & 4 & 2 & 118 & 58 & 48 & 0.67 & 0.78 & 0.19 & 0.12 & $0.1:$ \\
\hline
\end{tabular}

Table 3. Equivalence Checking Results

Versions column lists the version numbers of the artifacts compared. The Equiv column shows whether the versions are equivalent or not. The results contain data from three different configurations: (1) Full symbolic execution explores all paths, (2) iDiSE prunes paths that only differ in unimpacted constraints (iDiSE refers to the interprcoedural extension of the DiSE framework as defined in [16]), and (3) iSum represents the final impact summaries. The Paths column lists the number of paths, the Constraints column presents the number of constraints in the summaries, and Time Symbc column lists the time in seconds. The time reported for iDiSE includes the time to perform the static analysis and incremental symbolic execution. Finally, the Time Solver column lists the time taken by Z3 to solve the equivalence queries generated by full symbolic execution, iDiSE, and iSum. The rows marked with '-' indicate that the analysis does not finish within the time bound of one hour.

Overall, the results in Table 3 indicate that reducing the size of the queries reduces the time to check equivalence. In the tcas2 example, full symbolic execution generates 150 paths while iDiSE only generates 12 paths and we can see corresponding reductions in the number of constraints and time taken to check equivalence. The iDiSE overhead for the set of artifacts is quite small, and the total analysis time (Symbc + Solver) can be considerably less for iDiSE combined with constraint pruning over full symbolic execution. In the two versions of the kernel1 example, full symbolic execution is unable to complete the analysis within the time bound of one hour, while only four paths are gener- 


\begin{tabular}{|l|r|r||l|r|r|}
\hline Example & Modular & Non-modular & Example & Modular & Non-modular \\
\hline \hline tcas1V0V1 & 12.9 & 17.4 & tcas1V2V3 & 13.6 & 15 \\
\hline tcas1V1V2 & 13.6 & 15 & tcas2V0V1 & 14.3 & 18.2 \\
\hline wbs1V0V1 & 13.8 & 13.8 & wbs1V2V3 & 13.7 & 14.1 \\
\hline wbs1V0V2 & 13.8 & 13.8 & wbs2V0V1 & 14.6 & 14.4 \\
\hline replace2 V1V2 & 31.9 & $29: 53.2$ & & & \\
\hline
\end{tabular}

Table 4. Evaulation of artifacts using SymDiff

ated by iDiSE. There is a loop in kernel1 that does not contain any impacted statements; iDiSE is able to ignore paths through the loop and quickly generate the impact summaries. For this example, we can see how leveraging program similarities can dramatically improve the performance of regression verification. Although the time taken for equivalence checking for the other examples is relatively small - just a few seconds - the artifacts themselves are relatively small. We believe that the reductions will be applicable to larger examples as well. For the replace example, the solver time for the summaries without pruning is much faster than those with pruning. The tool we used to translate the CVC formula generated by KLEE into SMTLIB format (to be interpreted by Z3) parsed the CVC query into a trivial SMTLIB query for these examples. It is unclear to us why this occurred with the full summaries but not the impact summaries.

Limitations The regression verification technique presented in this work currently supports checking equivalence between two sequential programs without exceptional flow. The equivalence checking reports generated by Proteus are sound and complete for programs that do not have runtime errors or make calls to unsupported libraries. For examples that have runtime errors or make calls to unsupported libraries, the tool reports warnings and continues execution; the equivalence result are reported as inconclusive in the presence of such warnings. The sound and complete reasoning about the equivalence is with respect to a loop bound. It is possible to leverage automatic loop invariant generation and loop summarization techniques in the context of symbolic execution to reason about equivalent programs without a depth bound.

\section{Discussion}

Revisiting Table 3, the data in the Full columns can be considered representative of results in UC-KLEE [14]. The results demonstrate that UC-KLEE can benefit from using our reduction techniques, when analyzing related program versions.

We use SymDiff to perform equivalence checking on our artifact in order to evaluate SymDiff [9]. We set up the experiments for SymDiff and ran them on a Windows 7 machine with a $1.8 \mathrm{GHz}$ processor and $6 \mathrm{~GB}$ of RAM. We experimented with two SymDiff configurations (a) modular, where the methods are summarized as uninterpreted functions, and (b) non-modular, where the invocations to the different methods are inlined. The non-modular approach is sound and complete with respect to a depth-bound as well. The kernel and the 
cornell examples contain constructs that are not currently supported by the C front-end in the current version of SymDiff, so we report on experiments for the rest of the examples. Table 4 shows the total wall clock time in seconds. In the modular approach, SymDiff does not report any false negatives for the examples shown in Table 4. We used a loop bound of four for the replace example, the same as the one used in Table 3. We also used the flag in Symdiff to analyze only callers and callees that are reachable from the changed methods to ensure that the set of methods analyzed by SymDiff and Proteus is the same. SymDiff runs on a Windows platform while Proteus runs on a Unix-based platform; we had to run the experiments on different machines and it is not possible to make empirical comparative claims between the two in terms of time. Furthermore, SymDiff and Proteus encode the program behaviors differently, therefore, it is not possible to compare the approaches in terms of the size of the generated formulas. SymDiff does not use any slicing techniques based on change impact analysis, and we believe that it can be beneficial to add such a reduction technique to SymDiff.

Abstract Syntax Tree To calculate the precise initial change sets we can use standard algorithms to match Abstract Syntax Trees (ASTs), [13], and discard differences due to variable renaming and simple re-ordering before we perform the data and control flow analysis. The syntatic differences based on the ASTs are more precise compared to those generated by the GNU DiffUtils. We have support for the AST based syntatic diff in the Java implementation of the DiSE framework and we are currently working on adding it to Proteus.

Static Encoding vs. Bounded Unrolled Program Encoding The correctness of Eq. (1) does not rely on any specific encoding of constraints. We choose, however, to encode the program behaviors generated by symbolic execution (bounded unrolled programs) as constraints rather than use a static encoding for the constraints because (a) the static constraints on heap and array operations are often harder to solve than those generated by symbolic execution and (b) scalable static slicing techniques for interprocedural programs often ignore calling context and are imprecise; we leverage work in [16] to dynamically compute impact information for interprocedural programs.

\section{Related Work}

Several techniques have been developed for checking equivalence. Differential Symbolic Execution (DSE) uses uninterpreted functions to represent unchanged blocks of code [11]. SymDiff [9] summarizes methods as uninterpreted functions, and uses verification conditions to summarize observable behavioral differences. Regression verification techniques by Strichman et al. [7, 19] use the ContextBounded Model Checker (CBMC) to check equivalence of closely related C programs. It establishes partial equivalence of functions using a bottom-up decomposition algorithm. Another approach [18] performs an increment upgrade checking in a bottom-up manner similar to regression verification, using function summaries computed by means of Craig interpolation. These techniques are sound but not complete. Techniques from [18] are used in the PINCETTE 
project [4]. To curb over approximations, the PINCETTE project also employs dynamic techniques (e.g., concolic testing) to generate regression tests for system upgrades. There is also ongoing work to support program slicing based on the program differences in CBMC.

Similar to our work, UC-KLEE [14] is built on top of KLEE. UC-KLEE is designed to run two functions under test with the same input values and check if they produce the same outputs. As an optimization, UC-KLEE is able to skip unchanged instructions. However, it neither produces nor leverages the impacted behavior information. Partition-based regression verification, [2], computes partitions on-the-fly using concolic execution and dynamic slicing techniques. Each partition contains behaviors generated from a subset of the input space common to two program versions. The goal of the technique is to find test cases that depict semantic differences rather than prove equivalence.

Approaches that cache or reuse constraints to speed up performance (e.g., Green [20]) are orthogonal to our reduction technique. Such techniques are complementary to this work and can be leveraged to achieve higher reduction factors.

\section{Conclusions \& Future Work}

In this work on regression verification we leverage control- and data-flow information to partition the program behavior summaries as either impacted or unimpacted based on the differences between two program versions. We then prove that the impacted constraints of two closely related programs are functionally equivalent iff their entire program behavior summarizations are functionally equivalent. An empirical evaluation on a set of sequential $\mathrm{C}$ artifacts shows that reducing the size of the summaries helps reduce the cost of equivalence checking.

In future work, we plan to study the effects of other more compact program summarization encoding schemes such as large-block encoding [1] in combination with the work proposed here. Another avenue of future work is to develop an abstraction-refinement technique using uninterpreted functions to abstract large parts of the program as done in $[11,19]$, but, use the information about the impacted parts of the code to refine the abstraction when required. We believe such techniques can further improve checking equivalence of large programs.

Acknowlegements We thank Shuvendu Lahiri at Microsoft Research for his help with SymDiff.

\section{References}

1. D. Beyer, A. Cimatti, A. Griggio, M. Keremoglu, and R. Sebastiani. Software model checking via large-block encoding. In FMCAD, pages 25-32, Nov. 2009.

2. M. Boehme, B. C. d. S. Oliveira, and A. Roychoudhury. Partition-based regression verification. In ICSE, 2013.

3. C. Cadar, D. Dunbar, and D. R. Engler. Klee: Unassisted and automatic generation of high-coverage tests for complex systems programs. In OSDI, pages 209-224, 2008. 
4. H. Chockler, G. Denaro, M. Ling, G. Fedyukovich, A. E. J. Hyvrinen, L. Mariani, A. Muhammad, M. Oriol, A. Rajan, O. Sery, N. Sharygina, and M. Tautschnig. Pincette validating changes and upgrades in networked software. In CSMR, 2013.

5. L. M. de Moura and N. Bjørner. Z3: An efficient smt solver. In TACAS, pages 337-340, 2008.

6. V. Ganesh and D. L. Dill. A decision procedure for bit-vectors and arrays. In $C A V$, pages 519-531, 2007.

7. B. Godlin and O. Strichman. Regression verification. In $D A C, 2009$.

8. D. Kroening and O. Strichman. Decision Procedures: An Algorithmic Point of View. Springer Publishing Company, Incorporated, 2008.

9. S. K. Lahiri, C. Hawblitzel, M. Kawaguchi, and H. Rebêlo. Symdiff: a languageagnostic semantic diff tool for imperative programs. In $C A V$, pages 712-717, 2012.

10. C. Lattner and V. Adve. LLVM: A Compilation Framework for Lifelong Program Analysis \& Transformation. In CGO, 2004.

11. S. Person, M. B. Dwyer, S. Elbaum, and C. S. Păsăreanu. Differential symbolic execution. In FSE, pages 226-237, 2008.

12. S. Person, G. Yang, N. Rungta, and S. Khurshid. Directed incremental symbolic execution. In PLDI, pages 504-515, 2011.

13. S. Raghavan, R. Rohana, D. Leon, A. Podgurski, and V. Augustine. Dex: a semantic-graph differencing tool for studying changes in large code bases. In ICSM, pages 188-197, 2004.

14. D. A. Ramos and D. R. Engler. Practical, low-effort equivalence verification of real code. In $C A V$, pages 669-685, 2011.

15. G. Rothermel and M. J. Harrold. A safe, efficient regression test selection technique. ACM TOSEM, pages 173-210, 1997.

16. N. Rungta, S. Person, and J. Branchaud. A change impact analysis to characterize evolving program behaviors. In ICSM, 2012.

17. SAE-ARP4761. Guidelines and Methods for Conducting the Safety Assessment Process on Civil Airborne Systems and Equipment. SAE International, 1996.

18. O. Sery, G. Fedyukovich, and N. Sharygina. Incremental upgrade checking by means of interpolation-based function summaries. In FMCAD, UK, 2012.

19. O. Strichman and B. Godlin. Regression Verification - A Practical Way to Verify Programs. Springer-Verlag, Berlin, Heidelberg, 2008.

20. W. Visser, J. Geldenhuys, and M. B. Dwyer. Green: reducing, reusing and recycling constraints in program analysis. In SIGSOFT FSE, page 58, 2012.

21. W. Visser, K. Havelund, G. P. Brat, S. Park, and F. Lerda. Model checking programs. ASE, 10(2):203-232, 2003.

22. G. Yang, M. B. Dwyer, and G. Rothermel. Regression model checking. In ICSM, pages 115-124, 2009.

\section{A Theorem. 1 Proof}

Proof. We start by expanding the logical summation on the right hand side of the equivalence.

$$
\bigvee_{i=1}^{n}\left[\left(I_{i}^{0} \wedge U_{1}^{0}\right) \vee\left(I_{i}^{0} \wedge U_{2}^{0}\right) \vee \ldots \vee\left(I_{i}^{0} \wedge U_{n}^{0}\right)\right]
$$


The summaries of program version $V_{0}$ are symbolically represented by a disjunction of the possible paths through the program. Rules (1) - (4) in Table 1 dictate that unimpacted statements are not control dependent on any impacted statements. Therefore the conjunction of the constraints representing impacted statements on some path $i$ and the constraints representing unimpacted statements on some path $j$ are equivalent to the total constraints representing some path $k$. In other words, Formula 11 is valid.

$$
\bigvee_{i, j \in[1, n]}\left(I_{i}^{0} \wedge U_{j}^{0}\right) \leftrightarrow \bigvee_{i=1}^{n}\left(I_{i}^{0} \wedge U_{i}^{0}\right)
$$

As a consequence of Formula 11, the right hand side of Formula 10 simplifies to the left hand side of Formula 3.

$$
\bigvee_{i=1}^{n}\left[\left(I_{i}^{0} \wedge U_{1}^{0}\right) \vee\left(I_{i}^{0} \wedge U_{2}^{0}\right) \vee \ldots \vee\left(I_{i}^{0} \wedge U_{n}^{0}\right)\right] \leftrightarrow \bigvee_{i=1}^{n}\left(I_{i}^{0} \wedge U_{i}^{0}\right)
$$

\section{B Theorem. 3 Proof}

Proof. $(\Leftarrow)$ Assume Formula 13 is valid.

$$
\bigvee_{i=1}^{n}\left(I_{i}^{0} \wedge U_{i}^{0}\right) \leftrightarrow \bigvee_{i=1}^{m}\left(I_{i}^{1} \wedge U_{i}^{1}\right)
$$

From Formulas 4 and 5 we derive Formulas 14 and 15

$$
\begin{aligned}
& \forall_{x \in A I_{0}} \exists_{y \in A U}\left(I_{i}^{0}[x] \wedge U_{i}^{0}[y] \leftrightarrow I_{i}^{0}[x]\right) \\
& \forall_{z \in A I_{1}} \exists_{y \in A U}\left(I_{j}^{1}[z] \wedge U_{j}^{1}[y] \leftrightarrow I_{j}^{1}[z]\right)
\end{aligned}
$$

Furthermore, Formulas 14 and 15 yield Formulas 16 and 17

$$
\begin{aligned}
& \forall_{x \in A I_{0}} \exists_{y \in A U}\left[\bigvee_{i=1}^{n}\left(I_{i}^{0}[x] \wedge U_{i}^{0}[y]\right) \leftrightarrow \bigvee_{i=1}^{n}\left(I_{i}^{0}[x]\right)\right] \\
& \forall_{z \in A I_{1}} \exists_{y \in A U}\left[\bigvee_{j=1}^{m}\left(I_{j}^{1}[z] \wedge U_{j}^{1}[y]\right) \leftrightarrow \bigvee_{j=1}^{m}\left(I_{j}^{1}[z]\right)\right]
\end{aligned}
$$

Formula 13 is assumed valid, so any quantification over the program variables in Formula 13 is also be valid. This yields Formula 18 is valid.

$$
\begin{aligned}
\bigvee_{i=1}^{n}\left(I_{i}^{0} \wedge U_{i}^{0}\right) & \leftrightarrow \bigvee_{i=1}^{m}\left(I_{i}^{1} \wedge U_{i}^{1}\right) \\
& \rightarrow \\
\forall_{x \in A I_{0}} \forall_{z \in A I_{1}} \exists_{y \in A U}\left[\bigvee _ { i = 1 } ^ { n } \left(I_{i}^{0}[x]\right.\right. & \left.\left.\wedge U_{i}^{0}[y]\right) \leftrightarrow \bigvee_{i=1}^{m}\left(I_{i}^{1}[z] \wedge U_{i}^{1}[y]\right)\right]
\end{aligned}
$$

Formulas 16 and 17 yield Formula 19 


$$
\begin{gathered}
\forall_{x \in A I_{0}} \forall_{z \in A I_{1}} \exists_{y \in A U}\left[\bigvee_{i=1}^{n}\left(I_{i}^{0}[x] \wedge U_{i}^{0}[y]\right) \leftrightarrow \bigvee_{i=1}^{m}\left(I_{i}^{1}[z] \wedge U_{i}^{1}[y]\right)\right] \\
\forall_{x \in A I_{0}} \forall_{z \in A I_{1}} \exists_{y \in A U}\left[\left(\bigvee_{i=1}^{n} I_{i}^{0}[x]\right) \leftrightarrow\left(\bigvee_{i=1}^{m} I_{i}^{1}[z]\right)\right]
\end{gathered}
$$

Formulas 18 and 19 yield 20

$$
\begin{aligned}
& {\left[\bigvee_{i=1}^{n}\left(I_{i}^{0} \wedge U_{i}^{0}\right)\right.}\left.\leftrightarrow \bigvee_{i=1}^{m}\left(I_{i}^{1} \wedge U_{i}^{1}\right)\right] \\
& \rightarrow \\
& \forall_{x \in A I_{0}} \forall_{z \in A I_{1}} \exists_{y \in A U}\left[\left(\bigvee_{i=1}^{n} I_{i}^{0}[x]\right) \leftrightarrow\left(\bigvee_{i=1}^{m} I_{i}^{1}[z]\right)\right]
\end{aligned}
$$

The existential quantification over $A U$ can be removed from the right side of the implication because there are no unimpacted constraints on the right side. Furthermore, the universal quantification can be removed to maintain the validity of Formula 21.

$$
\left[\bigvee_{i=1}^{n}\left(I_{i}^{0} \wedge U_{i}^{0}\right) \leftrightarrow \bigvee_{i=1}^{m}\left(I_{i}^{1} \wedge U_{i}^{1}\right)\right] \rightarrow\left[\left(\bigvee_{i=1}^{n} I_{i}^{0}\right) \leftrightarrow\left(\bigvee_{i=1}^{m} I_{i}^{1}\right)\right]
$$

\section{Artifacts}

\begin{tabular}{|c||r|r|r|l|}
\hline Artifact & SLOC & Func & Description \\
\hline \hline Init_Data & 33 & 4 & $\begin{array}{l}\text { This is a C version of the example in Figure 4. } \\
\text { We note that we constrained the variable } \\
\text { capacity to be between 0 and 100. }\end{array}$ \\
\hline tcas & 173 & 9 & $\begin{array}{l}\text { Aircraft collision avoidance system } \\
\text { from Siemens Programs. }\end{array}$ \\
\hline replace & 564 & 21 & $\begin{array}{l}\text { Pattern matching and substitution program } \\
\text { from Siemens Programs. }\end{array}$ \\
\hline WBS & 214 & 1 & $\begin{array}{l}\text { Wheel Break System derived from WBS case } \\
\text { example in ARP 4761 and translated from Lustre. }\end{array}$ \\
\hline kernel & 133 & $6 \begin{array}{l}\text { Thread library supporting POSIX signaling } \\
\text { used to teach undergrad OS course. }\end{array}$ \\
\hline cornell & 302 & 12 & $\begin{array}{l}\text { Autonomous stability system for } \\
\text { a co-axial helicopter. }\end{array}$ \\
\hline
\end{tabular}

Table 5. Artifacts used in evaluation.

We evaluated our approach on six sequential $\mathrm{C}$ programs, including the Init Data example from Section 3.2, and examples from various domains and repositories. Table 5 presents details on each example. The examples can be found at the following locations:

- Init_Data: This is a C version of the example in Figure 4

- tcas: http://sir.unl.edu 
- replace: http://sir.unl.edu

- WBS: Rockwell Collins[17]

- kernel: https://facwiki.cs.byu.edu/cs345/index.php/Main_Page

- cornell: https://instruct1.cit.cornell.edu/courses/ee476/

FinalProjects/s2006/rg242/webpage/ece 476.htm

We created several versions using common refactoring patterns such as reversing or simplifying a conditional expression, reordering independent statements or blocks, simplifying calculations, removing syntactic sugar, among others. We also created versions that were incorrectly refactored in order to generate programs that are not functionally equivalent. We created test drivers to mark program variables of interest as symbolic and to invoke symbolic execution. Although we selected artifacts from various domains and manually created program versions using several common refactoring types, they may or may not be representative of $\mathrm{C}$ programs in general, or differences between program versions. We plan to evaluate our technique on larger benchmarks that have refactoring changes in their version history. 